\title{
Validation of Inlet and Exhaust Boundary Conditions for a Cartesian Method
}

\author{
Shishir A. Pandya * \\ NASA Ames Research Center, Moffett Field, CA \\ Scott M. Murman ${ }^{\dagger}$ \\ ELORET, Moffett Field, CA \\ Michael J. Aftosmis $\ddagger$ \\ NASA Ames Research Center, Moffett Field, CA
}

\begin{abstract}
Inlets and exhaust nozzles are often omitted in aerodynamic simulations of aircraft due to the complexities involved in the modeling of engine details and flow physics. However, the omission is often improper since inlet or plume flows may have a substantial effect on vehicle aerodynamics. A method for modeling the effect of inlets and exhaust plumes using boundary conditions within an inviscid Cartesian flow solver is presented. This approach couples with both CAD systems and legacy geometry to provide an automated tool suitable for parameter studies. The method is validated using two- and three-dimensional test problems which are compared with both theoretical and experimental results. The numerical results demonstrate excellent agreement with theory and available data, even for extremely strong jets and very sensitive inlets.
\end{abstract}

\section{Introduction}

Simulation of external aerodynamics is an important aspect of the aircraft design cycle. While the present simulation capability is often adequate for prediction of forces and moments on the vehicle, the inlets and exhaust nozzles are usually faired over to avoid complexity. This simplifying approach makes an implicit assumption that power effects play a minor role in establishment of the vehicle aerodynamics. In practice this assumption often leads to discrepancies between the simulation and flight vehicle that necessitate aposteriori correction of the simulation results. This lack of correlation between flight and simulation limits the use of computational modeling as a predictive tool.

The assumption that power effects are minimal breaks down in a variety of well known circumstances. For example, the presence of an inlet that draws flow in or an exhaust with a substantial plume can have a noticeable effect on the flow field[1]. Flow features such as the thickness of the boundary layer, shock location and strength of a shock can be altered due to the presence of a plume. In both visocus and inviscid simulation, the lack of a plume may effect the forces and moments on the vehicle. Examples include a jet-plume-induced flow separation on the surface of the vehicle [2] and the changes in the flow in the rear part of the vehicle due to the presence of an under-expanded plume [3]. Studying the effects of the plume flow on the control surfaces in the empannage and the resulting changes in pitching and yawing moments can also be important for determining the stability characteristics of the aircraft.

In cases where the inlet and exhaust effects are important, an engineering approximation that captures the gross aerodynamic effects without the complexity of modeling an engine or the chemistry in the plume

*Aerospace Engineer, Member AIAA

${ }^{\dagger}$ Senior Research Scientist, Member AIAA

$\ddagger$ Aerospace Engineer, Member AIAA 
is needed. Such an engineering method can be adequate if the inlet/exhaust flow conditions are known and when the plume chemistry is not expected to have a large effect on the flow. This need for an engineering tool is addressed here with an inlet boundary condition at a compressor face within an inlet duct or at an upstream location within a nozzle.

In the current work, the ability to simulate inlet ducts and exhaust plumes is added to the Cartesian mesh-based aerodynamic simulation package Cart3D[4]. The Cart3D package consists of a set of tools for automatically and efficiently generating a Cartesian volume mesh with cut-cell boundaries from component triangulations. The flow solver within the Cart3D package is a scalable, parallel, inviscid solver for a Cartesian $\operatorname{mesh}[5,6]$.

The implementation consists of a component-based approach where each triangle in the input geometry is assigned a component identification tag. These component IDs can be assigned a boundary condition and a reference state in the flow solver. The flow solver is modified to use this information to produce the appropriate inlet or exhaust behavior at the given boundary using a characteristics-based approach. To implement the boundary condition the effected Cartesian cells are identified, and the Riemann problem is solved at the boundary-faces of these cells with the user specified boundary state.

In this work, the implementation of the boundary conditions is first validated by comparison to analytical solutions for inlets and then by comparison to experiments for exits. A simple test case to verify that the correct inlet behavior is obtained is a Pitot type inlet. The Pitot inlet can be modeled for comparison with an exact solution for several conditions. A wedge-shaped diffuser for which the flow conditions can be computed based on the normal and oblique shock relations is a more challenging inlet case where the exact shock angles must be recovered in order to obtain the proper inlet behavior. The solution of an F-16 inlet is also obtained to illustrate the application of the method to a complex geometry.

Exhaust cases are demonstrated next. The Future European Space Transportation Investigation Program (FESTIP) test case [7] is used to verify that the gross features of the flow field are accurately recovered. The solution is compared to an experimental Schleiren photograph. The simulation of a tangent-ogive missile body is compared to experiment[8] to demonstrate the use of the boundary conditions on an exhaust case where the exhaust has an effect on the vehicle aerodynamics. An under-expanded plume test case, for which the pressure ratio of the plenum to the free stream is extremely high, is shown for validation at extreme conditions. The Space Shuttle in ascent configuration with plumes emanating from the main engines (SSME) and solid rocket boosters (SRB) is also computed to further show the utility of the tool in "real-world" scenarios with complex geometry.

\section{Method}

An inlet or exhaust in the unstructured Cartesian flow solver is modeled using the following steps. First, subsets of the triangulation of the intersected geometry are marked as inlet or exhaust faces. These faces are identified by using component IDs and reference conditions are assigned for these faces by the user. The second step consists of marking any Cartesian cells which intersect any marked triangle. The third step uses a Riemann problem to apply a boundary condition on the boundary faces of these Cartesian cells. The solution of the Riemann problem determines the conditions in the Cartesian cells next to each marked triangle resulting in either an inlet or an exhaust. Each of these steps is described in detail in this section.

\section{A. Marking the mesh}

Cart3D relies on a component-based approach where each component (e.g. wing, fuselage, etc.) is triangulated separately and assigned a component identification tag. The final configuration is generated by intersecting the component triangulations resulting in a single, water-tight triangulation of the wetted surface. Component numbers are preserved so that each triangle retains its original component ID. Thus, the component number can be associated with each part of the surface geometry (e.g. wing, fuselage, vertical tail). The preferred approach is to assign component IDs at the CAD level and propagate the component 
information through the mesh generation process. However, in order to handle legacy geometry it is also necessary to mark subsets of water-tight triangulations directly.
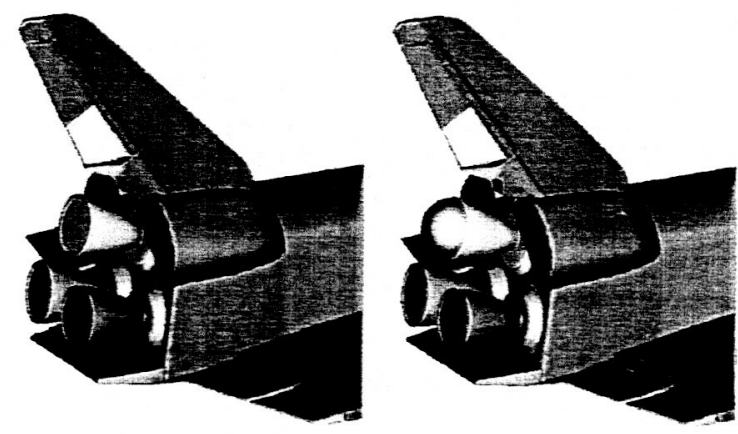

(a) Marking using a sphere

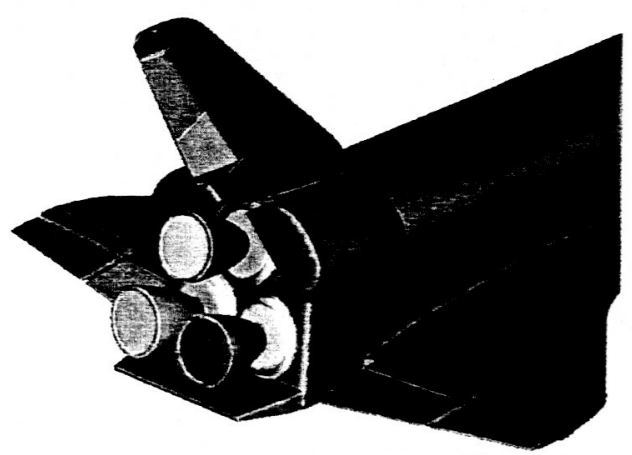

(b) Exit planes marked(shown by color)

Figure 1. Marking the triangles as inlet or Exit

The component-based approach is extended in the current work so that a subset of a water-tight triangulation can be labeled as an inlet or exhaust. Two methods are presently available for marking a water-tight triangulation. The first method uses a "cookie-cutter" (e.g. a bounding box, a sphere) to specify a region. All triangles which lie within this region are marked as inlet/exhaust. The second method uses a painting algorithm. In this approach, a triangle on the inlet or exhaust face is picked by the user and the neighboring triangles are marked until the boundary of the current triangle makes a large angle (user specified) with its neighbor. In this manner, planar or smoothly varying surface patches can be marked from a single initial "seed" point.

Figure 1(a) shows an example of the method where a sphere is used to assign exhaust planes on the back end of a Space Shuttle orbiter's main engines. The user specifies a sphere as shown in Fig. 1(a) to extract the exhaust plane of the top nozzle as a separate component. Figure 1(b) shows the resulting component marking. Components are distinguished from each other by colors.

When the Cartesian mesh is generated by Cart3D, the triangulation is intersected with the Cartesian cells forming a set of cut cells around the surface of the geometry. These cut cells are arbitrary polygons in $2 \mathrm{D}$ and polyhedra in $3 \mathrm{D}$. Each cut-cell may intersect portions of one or more triangles. If any of the triangles in the cut-cell are marked inlet/exhaust, the cut-cell is also marked so that the flow solver will use the Riemann invariants-based boundary condition to model the appropriate behavior.

\section{B. Flow solver algorithm}

In order to solve the Riemann problem at a boundary of a cut-cell marked for inlet/exhaust, the user must specify a reference state that corresponds to the flow condition inside this boundary. Such a cell is depicted in Fig. 2 where the user-specified flow conditions inside the boundary are denoted $\overrightarrow{Q_{L}}=\overrightarrow{Q_{\text {ref }}}$. The flow conditions in the cut-cell in the domain adjacent to the boundary are reconstructed from the flow variables in the local neighborhood and the reconstructed state vector is denoted $Q_{R}$.

$$
Q_{R}=Q_{i}+\vec{r} \Delta Q_{i}
$$

where $Q_{i}$ is the state vector in the Cartesian cell next to the boundary.

While the mesh is Cartesian, the Riemann problem at the cut cells is solved in a non Cartesian-aligned plane. Therefore, the velocity sent to the Riemann solver must be rotated into the coordinate system aligned 
with the normal to that boundary face.

$$
U_{n}=\vec{U} \cdot \hat{n}
$$

where the subscript on $\vec{U}$ can be either $U_{L}$ or $U_{R}$, and $\hat{n}$ is the unit normal vector.

Having rotated the velocity vectors, a Riemann problem can be solved to compute the flux across that piece of the cut cell using any of the flux functions, $f$, available in the solver.

$$
F=f\left(Q_{L}, Q_{R}\right)
$$

The solution of the Riemann problem has the benefit that the user need not supply whether the present boundary is an inlet or an exhaust. This determination is a result of the Riemann problem based on $Q_{L}$ and $Q_{R}$ and the Riemann solver will take the appropriate characteristic information from the boundary state.

At a supersonic exhaust, the result is that the boundary reference state specified by the user becomes the state for that boundary face. At a supersonic inlet, the Riemann solver takes all characteristics from the flow next to the boundary. For subsonic flow, the Riemann solver uses the flux function being used by the solver to compute the new flux based on $Q_{L}$ and $Q_{R}$.

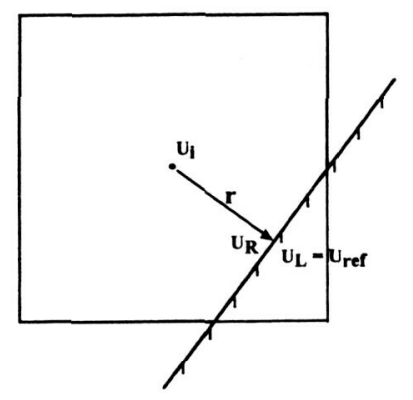

Figure 2. A Cartesian cell is cut by a boundary forming a cut cell.

ck to the original frame of reference and then added to the appropriate flux.

$$
F=F_{n} \hat{n}+F_{\tau} \hat{\tau}
$$

The computation of the flux is in large part dependent on the choice of the user specified reference state. The wrong choice can result in an incorrect solution of the Riemann problem resulting in erroneous flow behavior. Additionally, the present tool makes it possible for a user to specify the boundary conditions at one of several locations. One approach may be to specify the conditions at the inlet or exit plane. The advantage of this approach is that the details of a nozzle duct or inlet cowl do not need to be modeled making the geometry much simpler. However, the conditions at such a face are rarely constant along the boundary face making the choice of the reference state challenging. For an exhaust case for example, the flow at the exit plane depends on the shape of the sonic surface in the throat. Therefore, it is difficult to specify the flow conditions at the exit plane. The preferred approach, therefore, is to model the details of the nozzle and specify the total temperature and pressure in the plenum. This latter approach is used in the present work.

\section{Results}

The implementation of these boundary conditions is validated using several test cases and the results are compared to an analytic solution, or experimental data. The inlet verification and validation cases are presented first, followed by exhaust validation.

Supersonic intake design makes use of carefully controlled shock-systems to process the air that enters the inlet. For this reason, two supersonic intake computations are compred to theoretical results for verification of the method. A classical Pitot intake is used to show that the method is capable of predicting the correct shock behavior for a variety of back pressures. For a more complex shock system, a wedge-shaped diffuser is modeled, where an oblique shock off the lip of the wedge slows the flow initially and a strong shock at the lip of the cowl reduces the flow speed to subsonic. Lastly, the flow around an F-16 fighter aircraft with an active inlet is modeled to illustrate the utility of the method. The results are compared to experment for validation.

Following the inlet results, three exhaust validation test cases are presented followed by a Space Shuttle in the ascent configuration. The FESTIP geometry with an under-expanded plume is computed and compared

4 of 16

American Institute of Aeronautics and Astronautics 
to an experiment. A tangent-ogive missile body at $M=0.9$ for which the plume effects the pressure on the rear part of the missile body is simulated for several plume shapes. The plume shape is controlled by the pressure in the plenum chamber. The results are compared to experimental pressure data. A hypersonic free jet is also computed and the results are compared to experiment validating the usefulness of the capability for very large plenum to free stream pressure ratios. Finally, the full Space Shuttle launch vehicle with all the details of attachment hardware, etc. is modeled with plumes to demonstrate a practical application of the method.

\section{A. Pitot intake}

The Pitot intake can often be attractive to a designer due to its low drag. It is also an attractive two dimensional test case for verification as its behavior is well understood. The behavior of the Pitot intake is depicted in fig. 3 for the critical condition. If the inlet is placed in a $M_{1}=1.4$ flow, specifying the flow conditions in the inlet tube $\left(M_{B}, P_{B}, \rho_{B}\right)$ using the Rankine-Hugoniot relations requires that the air be processed by a normal shock (depicted in red at the mouth of the inlet). Since the Pitot tube is essentially a control volume, and these conditions provide identical mass, momentum and energy flux in a constant area stream-tube, this set of conditions define the critical condition for the Pitot tube. These conditions $\left(M_{B}=0.7397, \frac{p_{B}}{p_{1}}=2.1199, \frac{\rho_{B}}{\rho_{1}}=1.6896\right)$ can be used for specification at the boundary face (depicted in green). From the critical case, conditions for sub and super-critical cases may

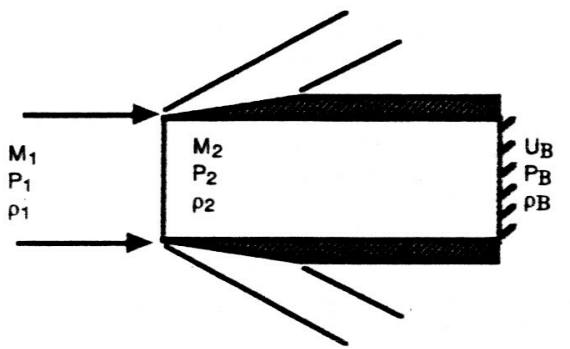

Figure 3. The Pitot intake: Shocks are depicted in red with the normal shock corresponding to the critical condition. be obtained by increasing or decreasing the back-pressure of the inlet $\left(P_{B}\right)$ to force spillage, or partial shock swallowing.

Based on the critical condition, the boundary conditions are tested on four scenarios shown in Fig. 4. In case one, the intake is blocked. The flow can not go through the intake. This results in a bow shock well ahead of the intake. In case two (sub-critical), there is flow through the intake, there is still some spillage resulting in a bow shock closer to the intake lip. The third case, termed critical, does not let flow spill around the lip. This results in a normal shock just at the lip. This condition is also called the maximum flow condition. In the fourth case (super-critical), the pressure in the channel is lower than the critical case resulting in a delayed shock. Thus, the shock occurs well inside the intake channel.

The computation of these cases is performed on a Cartesian mesh refined around the intake channel to capture the

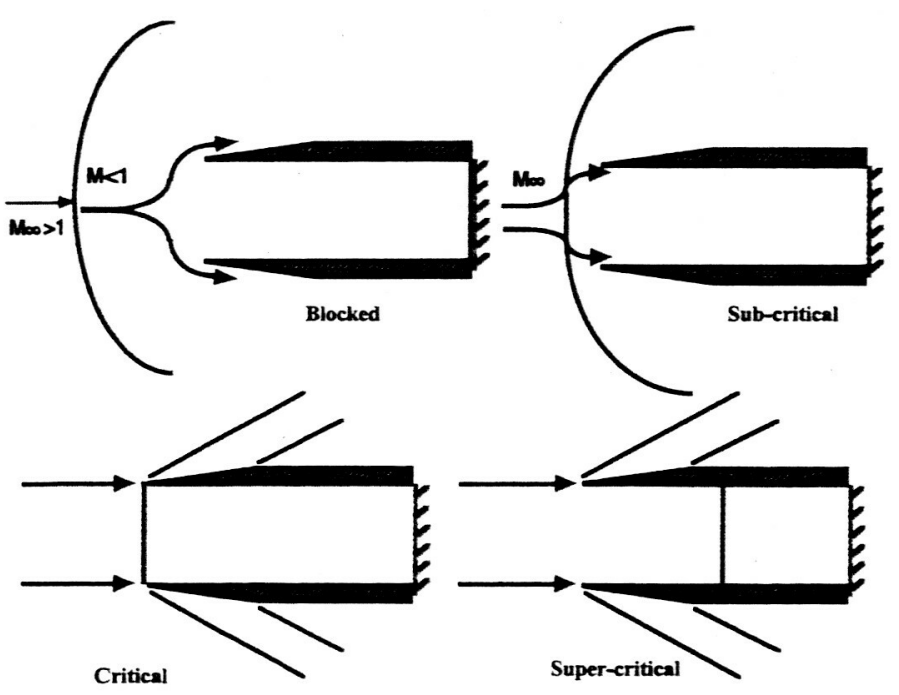

Figure 4. The Pitot intake: Depicted are the four states at which the boundary conditions are tested. shocks properly. Figure $5(\mathrm{a})$ shows pressure contours from a computation of the case where the intake is blocked. For this case, the boundary 
conditions are specified to be zero velocity. It can be seen that a bow shock well ahead of the intake lip has formed as expected and the particle traces do not enter the inlet.

The pressure contours for the sub-critical case are shown in fig. $5(b)$ for $\frac{p_{B}}{p_{1}}=1.7$. Here a bow shock is seen just ahead of the intake lip. The particle traces verify that air is flowing through the intake, but some air spills to the outside. A higher than critical pressure is used to specify the boundary conditions for the sub-critical case.

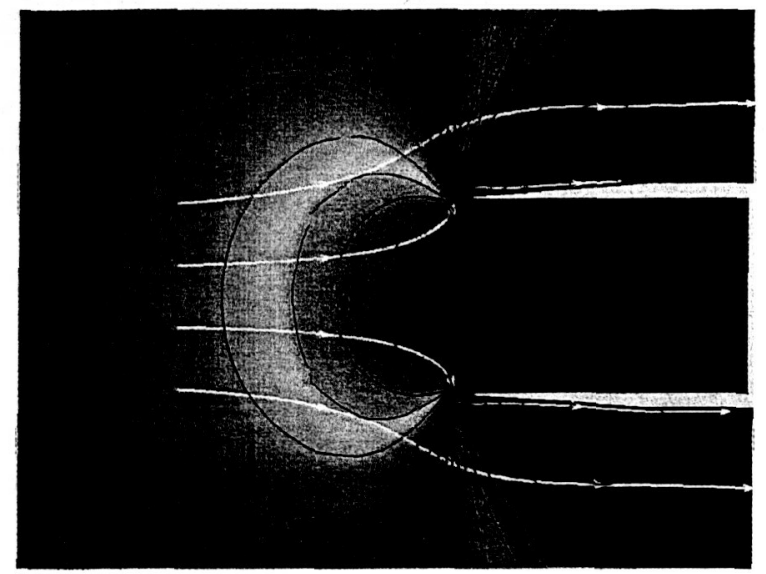

(a) blocked

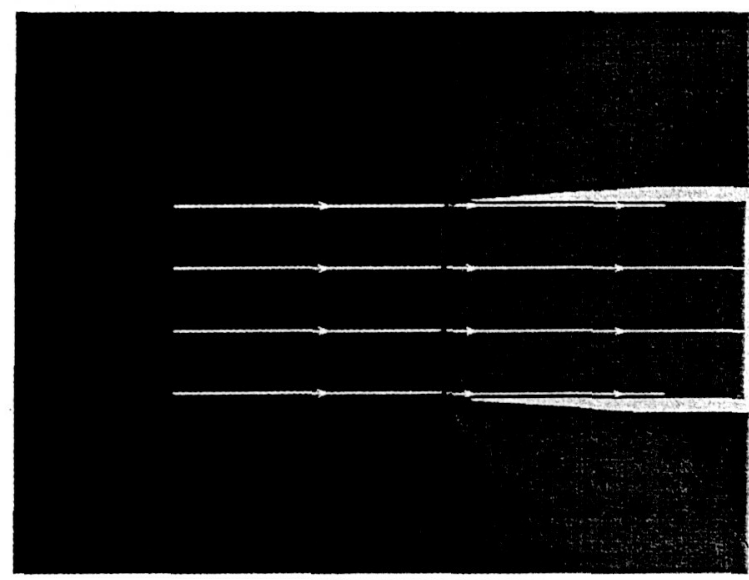

(c) critical, $\frac{p_{B}}{p_{\infty}}=2.1199$

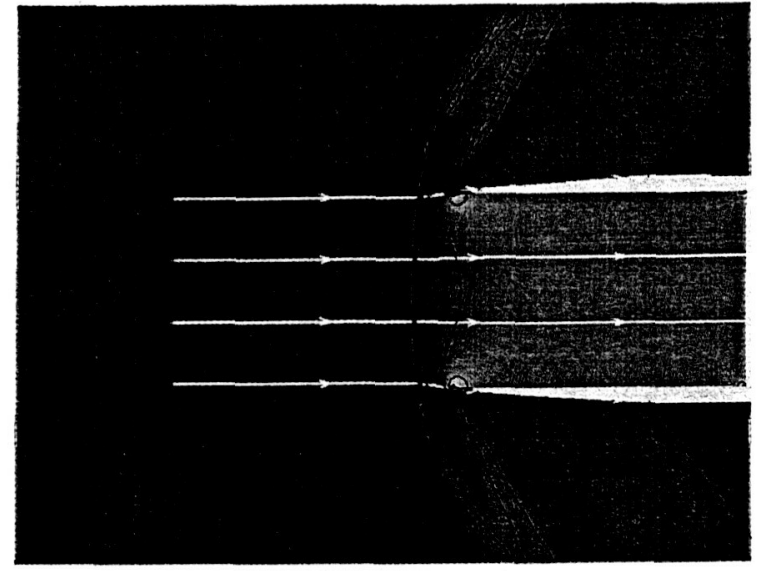

(b) sub-critical, $\frac{p_{B}}{p_{\infty}}=1.7$

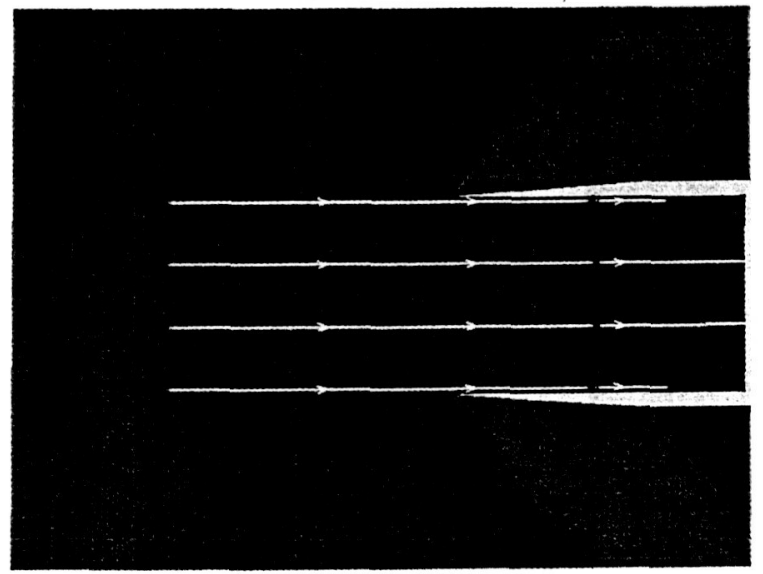

(d) super-critical, $\frac{p_{B}}{p_{\infty}}=2.54$

Figure 5. The Pitot intake at $M_{\infty}=1.4$

The pressure contours for the critical case are shown in fig. 5(c). Here a normal shock forms at the intake lip as expected. To verify that the maximum flow condition has been achieved, particle traces are shown. The particle traces verify that there is no spillage. The critical conditions are specified for this test case based on theory and are discussed above.

The pressure contours for the super-critical case are shown in fig. $5(\mathrm{~d})$ for $\frac{p_{B}}{p_{1}}=2.54$. Here the normal shock is seen inside the intake channel. The particle traces verify that no air spills to the outside of the lip. A lower than critical pressure is used to create the super-critical conditions. 


\section{B. Two-shock wedge diffuser}

A 2-shock wedge-shaped inlet, depicted in fig. 6, is modeled in two dimensions to show that the method accurately predicts the effect of the inlet conditions. This is an especially demanding test case because it depends on being able to model precise shock impingement so that the flow stays parallel to the wedge surface. The inlet is designed to turn the flow through the first shock (an oblique shock) such that the flow behind the shock is parallel to the ramp. Mis-predicting the shock strength results in the first (weak) shock missing the upper lip and thus never setting up the second shock. If the correct shock strength is obtained, a strong shock at the cowl lip forms, turning the flow parallel to the axis of the inlet and slowing the flow to subsonic speeds. The strong shock impinges precisely at the lower wall corner so that the flow is turned only by the shock. Missing any of these details results in an erroneous solution which takes the form of either a system of reflecting oblique shocks in the duct or a single detached weak shock. As with the critical Pitot inlet case, flow at the inlet face where the boundary state is specified is subsonic.

The exact solution to the flow in this inlet can be derived using the oblique shock tables for the first shock[9]. For a free stream speed of $M_{\infty}=2.0$, the pressure and density jumps across the weak shock are 1.3154 and 1.2156 respectively with the speed behind the shock corresponding to $M=1.82125$. The Mach number behind the strong shock (0.61965) for a turn angle of $5^{\circ}$ is read from the strong shock branch of the

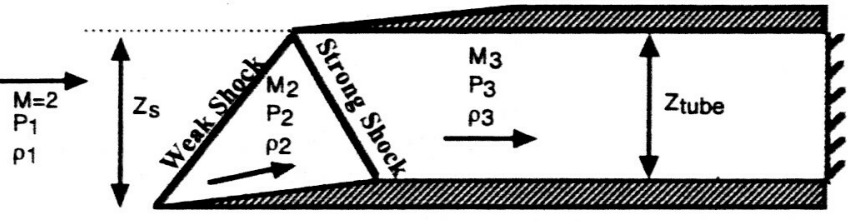

Figure 6. The two shock Wedge diffuser oblique shock charts and corresponds to a shock angle of $86.36^{\circ}$. The corresponding pressure and density jumps are 3.6875 and 2.3871 respectively.

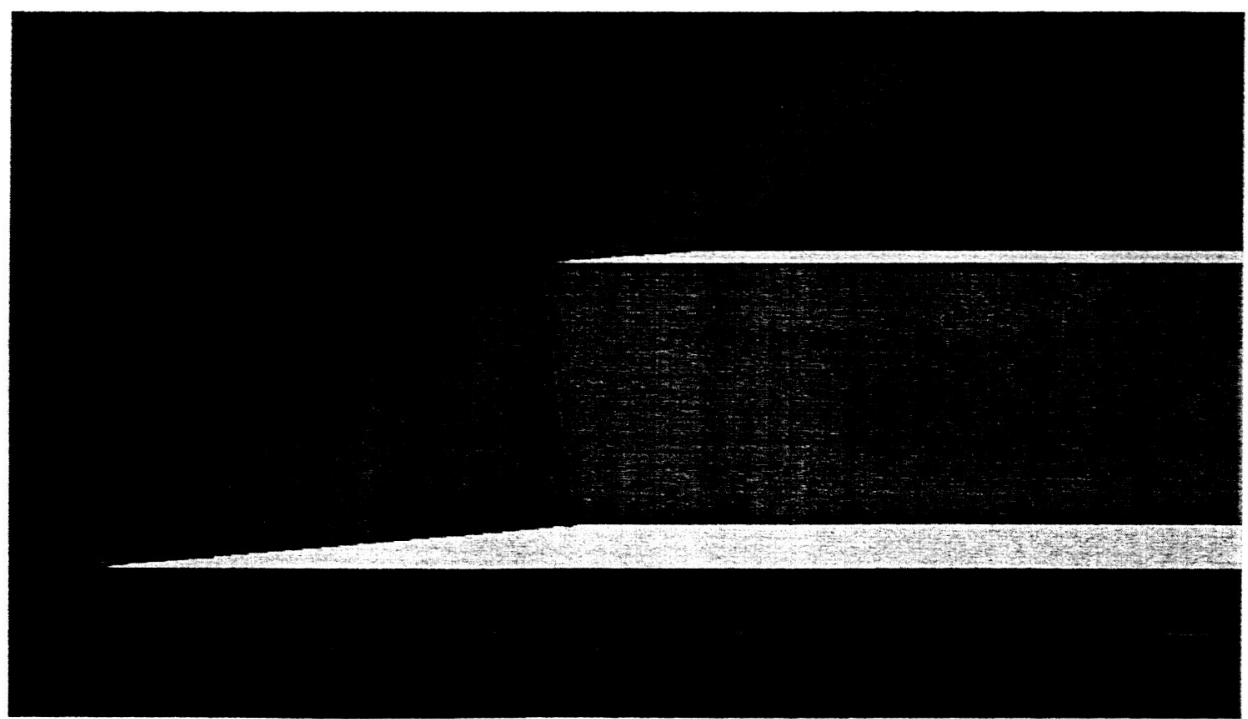

Figure 7. Pressure contours for the two shock Wedge diffuser

A geometry of the $5^{\circ}$ wedge and inlet duct that corresponds to the maximum flow condition is created. The area of the inlet is computed using the conservation relation,

$$
\rho_{1} u_{1} Z_{s}=\rho_{3} u_{3} Z_{\text {tube }}
$$


where $Z_{s}$ is the capture area, $\rho_{1}$ and $u_{1}$ correspond to the free stream conditions, and $\rho_{3}$ and $u_{3}$ correspond to the density, and speed in the tube of size $Z_{t u b e}$. For the present test case, $Z_{s}=0.2069$ and $Z_{t} u b e=0.17799$ for a $5^{\circ}$ wedge and a wedge length of 0.4 .

The resulting flow field is shown in figure 7 . With a back pressure of 4.8505 and back density of 2.9017 , the computed solution is in excellent agreement with the exact solution.

\section{F-16}

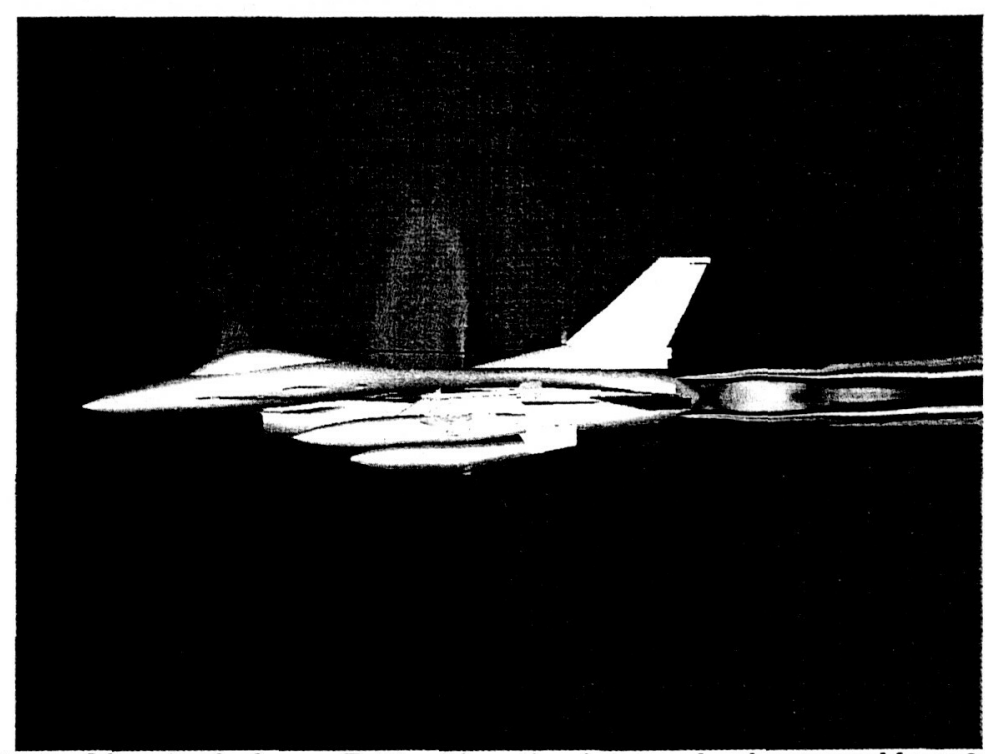

Figure 8. Velocity Magnitude for an F-16 with active inlet and exhaust at $M_{\infty}=0.85$ and $\alpha=16^{\circ}$

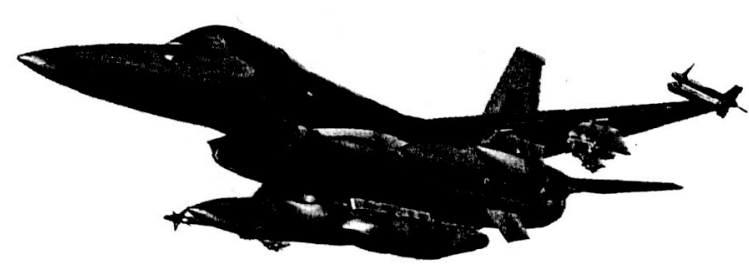

(a) Contours, cut shown in black

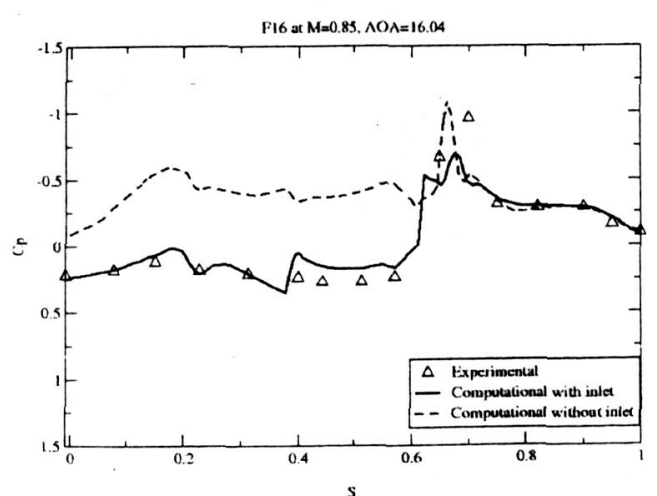

(b) $C_{p}$ along the cut

Figure 9. Coefficient of pressure for the F-16 at $M_{\infty}=0.85$ and $\alpha=16.04$

The present method can be easily applied to a vehicle with complex geometry. An F-16 is simulated with an active inlet as well as an active exhaust for demonstration. Specialized methods with the intent 
of capturing the effects of an active inlet on the pressure close to the inlet duct have been used for F-16 simulation in the past $[1,10]$. Based on these, a set of reference conditions that do not modify the conditions at the inlet are specified. A plume is simulated with a high velocity exhaust.

Figure 8 shows the flow field around the fighter aircraft at $M_{\infty}=0.85$ and $\alpha=16.04^{\circ}$. At the inlet face, the reference Mach number is set to 0.75 and at the exhaust, the reference Mach number is set to 2.2 leaving the pressure at both equal to the upstream ambient pressure. In this figure, the colors represent the speed of the flow. Stream traces close to the inlet duct show flow entering the inlet as well as going around the inlet. The effect of the flow into an active inlet can be seen in the change in pressure in the vicinity of the inlet cowl. Figure 9(a) shows contours of the pressure coefficient on the surface of the vehicle. A black line in the figure represents the circumferential cut along which values of the pressure coefficient are compared in Fig. 9(b) with and without inlet modeling. $S$ in the plot is the arc length along the cross section of the fuselage starting at the bottom. The difference in these pressure distributions affirms the need for modeling an active inlet. The pressure coefficient in Fig. 9(b) is also compared to experiment[10] and agrees closely. The slight discrepancy in the pressure near $S=0.4$ may be due to the addition of the gun-port geometry for the M61 A1 Vulcan $20 \mathrm{~mm}$ gatling gun system in the present simulation.

\section{FESTIP}

In addition to being able to predict the inlet flows correctly, the method also allows accurate prediction of the effects of an exhaust plume. We first focus our attention on the qualitative features of the flow field with the plume. In order to verify that we obtain a properly sized plume and that we are able to capture the effects of the plume on the flow field, the FESTIP vehicle is simulated at Mach 2.98 with plume conditions specified [7]. The resulting flow field is shown in fig. 10(b) and is compared to a Schlieren photograph (fig. 10(a)) from the experiment. The comparison shows that the present method correctly predicts not only the bow shock structure due to the vehicle and sting, but also correctly captures the extent of the plume, and compression shock which forms near the exhaust.

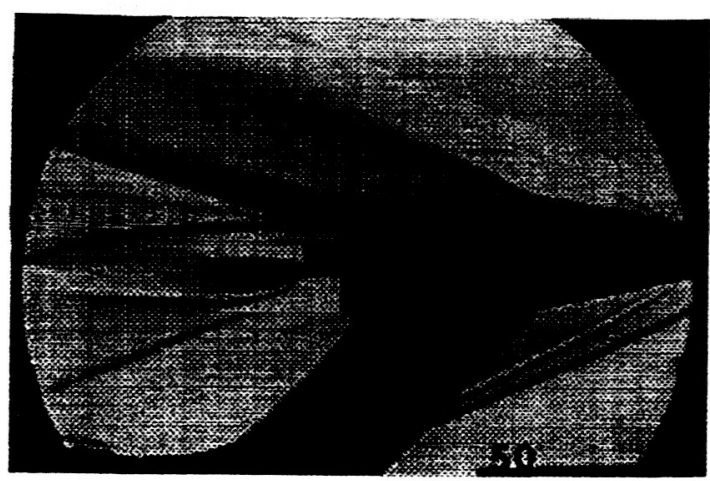

(a) Experimental

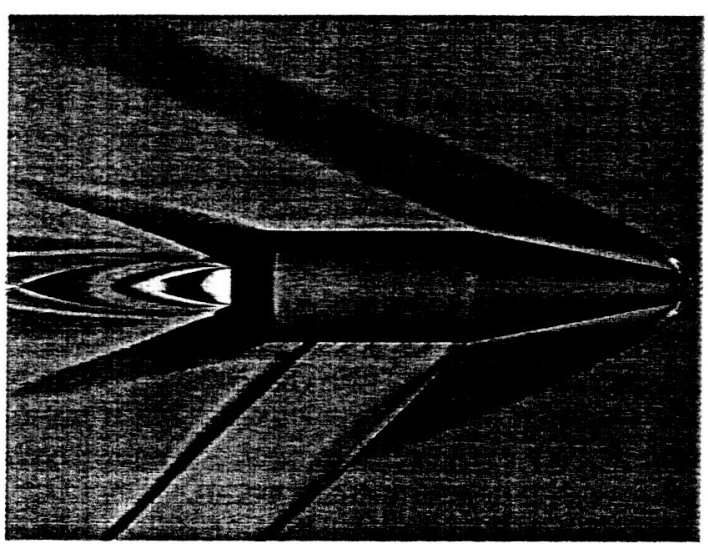

(b) Computational

Figure 10. Density contours on the surface of the FESTIP and the center plane at $M_{\infty}=2.98$ compared to experiment [7]

\section{E. Tangent-ogive plume}

A plume often has an effect on the flow near the aft-body area, where flow turning to accommodate the plume can put a shock on the aft part of the vehicle, drastically modifying the surface pressure and velocities and thus effecting forces and moments. This effect is especially noticeable for under-expanded plumes. Thus, 
a quantitative validation of the plume capability of the boundary conditions can be performed by examining the pressure variation on the body with the size of the plume. An ogive-shaped missile for which the effect of the plume on the surface of the aft-body is experimentally documented for several plume conditions is chosen.
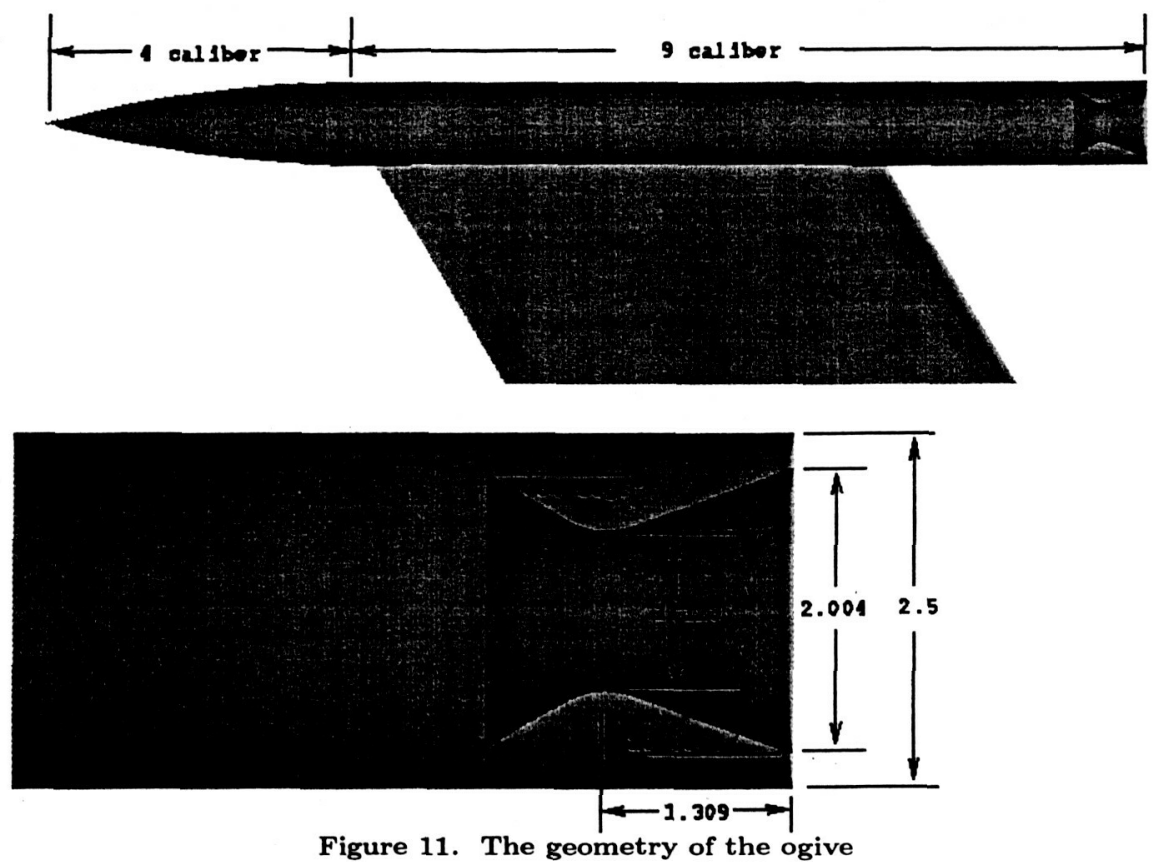

A strut-mounted body of revolution (see Fig. 11) with a cylindrical after-body is used in an experiment by Burt [8] at $M=0.9$ and $M=1.2$ with zero angle of attack. This case has been more recently computed by Raghunathan et. ai [11] using a viscous technique. The model has a 4-caliber tangent-ogive nose attached to a 9-caliber cylindrical body. A $20 \mathrm{deg}$. conical nozzle with a design Mach number of 2.7 is modeled to match the experiment.

Conditions are specified at the vertical face in the plenum chamber which correspond to the experimental conditions, including the pressure ratio between the plenum and the free stream. The specification of the pressure in the plenum provides the mechanism by which the air is pushed through the throat and the nozzle. The resulting plume is depicted by Mach number contours in Fig. 12 where blue denotes low speed flow such as that in the plenum, white denotes the high speed flow at approximately Mach 7 and the colors in between denote intermediate values.

The pressure on the surface of the cylindrical after-body is reported by the experiment $[8]$ for a range of pressure ratios. The lowest pressure ratios correspond to an over-expanded plume while high pressure ratios correspond to an under-expanded plume. A blockage to the main flow develops as a result of the under-expanded plume. The plume grows larger with higher pressure ratios. Thus, at high pressure ratios the plume has a larger effect on the aerodynamics in the region of the cylindrical after-body. This effect can be seen by examining the changes in pressure on the after-body. The pressure on the after-body is therefore compared to the experiment in Fig. 13(a) and shows good agreement in both trend and value.

A similar plot on the same body for free stream Mach number of 1.2 is also shown in figure 13(b). For this supersonic case, the vehicle develops a shock on the cylindrical after-body due to the blockage from the under-expanded plume. Due to the boundary layer development, the compression in the experiment is not 

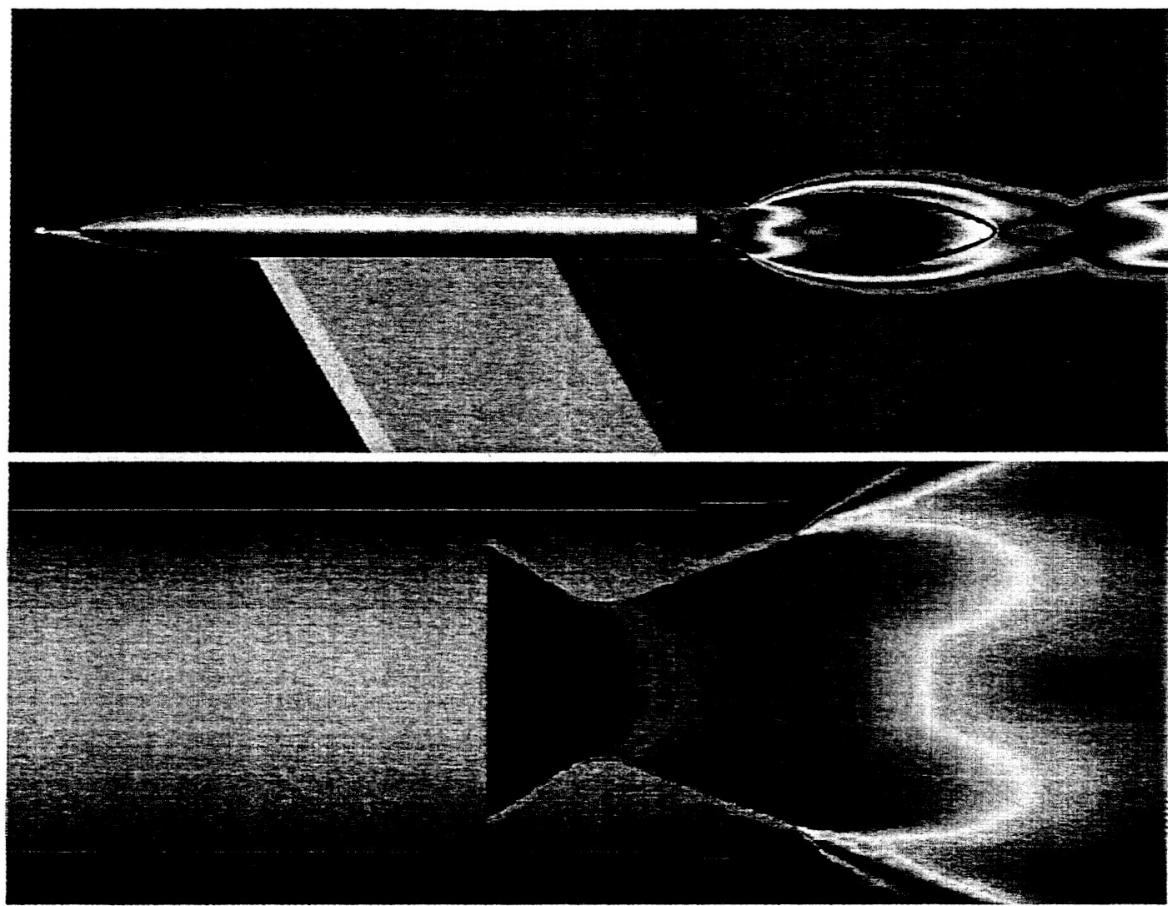

Figure 12. The pressure contours behind the ogive showing the plume

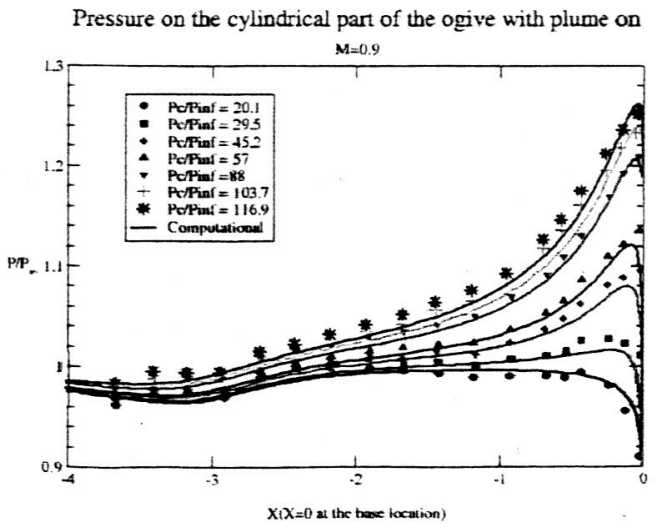

(a) $M_{\infty}=0.9$

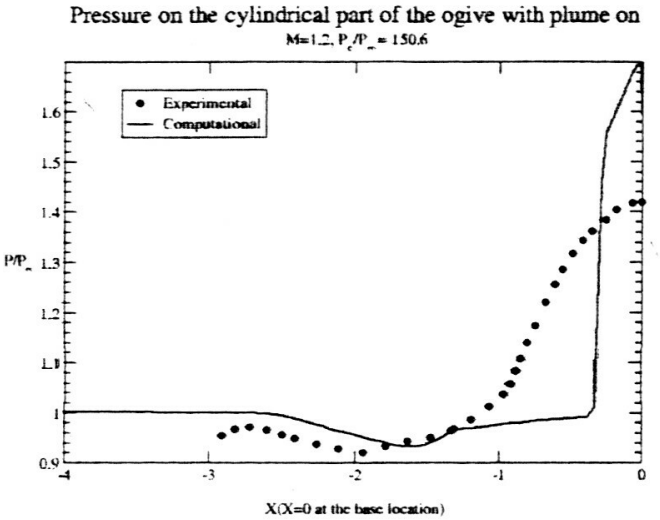

(b) $M_{\infty}=1.2$

Figure 13. Pressure on the surface of the ogive compared to Experiments $[8](x=0$ is the base of the ogive body) 
as abrupt as the inviscid simulation and as a result, the shock location is not well-predicted by the solution of the Euler equations, but the effect on the moment is similar.

\section{F. Under-expanded jet}

Table 1. Flow conditions upstream of the orifice for the under-expanded jet

\begin{tabular}{|c|c|c|c|}
\hline case & $P_{T}$ & $T_{T}$ & $P_{\text {back }}$ \\
\hline 1 & $1.3 \mathrm{~atm}$ & $297 \mathrm{~K}$ & $2.68 \times 10^{-4} \mathrm{~atm}$ \\
2 & $1.13 \mathrm{~atm}$ & $297 \mathrm{~K}$ & $1.37 \times 10^{-4} \mathrm{~atm}$ \\
\hline
\end{tabular}

A hypersonic, under-expanded, free jet, such as one emanating from a reaction control system (RCS), is a challenging problem due to extremely large pressure ratios producing a large under-expanded plume and the presence of a strong shock. The jet is released from a small orifice into near-vacuum conditions such that the ratio of the plenum pressure $\left(P_{T}\right)$ to the ambient flow is approximately 3500 . This severe change in conditions results in a jet that accelerates to Mach 16 and has gradients in radial as well as axial directions [12]. The jet is terminated by a strong shock which brings the flow from $M=16$ to subsonic speeds. The experiment was performed at two sets of conditions which are given in table 1.

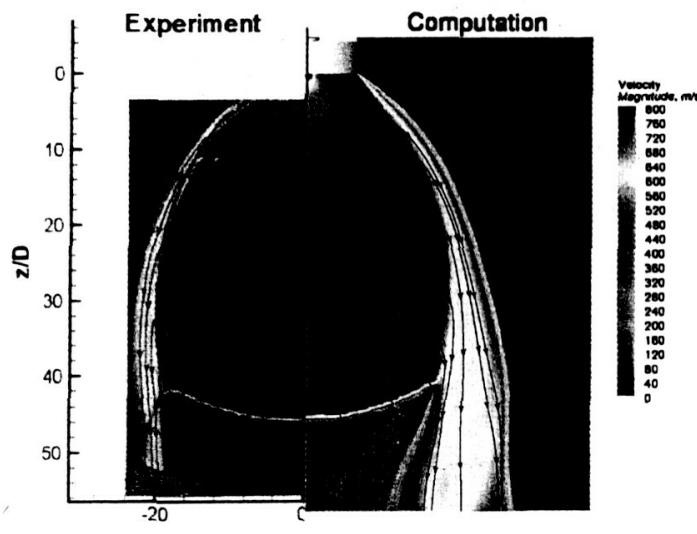

(a) Global velocity contours

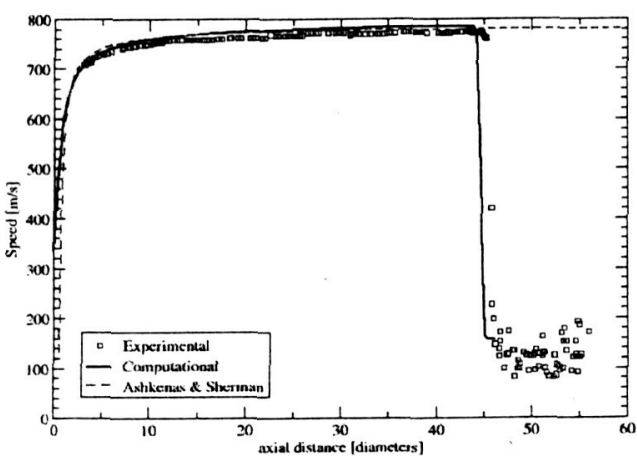

(b) Speed along the centerline

Figure 14. Velocity comparisons for an under-expanded free jet (case 1)

A plot of the velocity magnitude is shown for case 1 in fig. 14(a) and compared to planar laser induced iodine fluorescence (PLIIF) measurements from experiment [12]. The bell-shaped plume is visible with the strong shock at the edge of the plume. Both the shape of the plume and the location of the shock agree extremely well with experiment and are similar to the computational results presented by McDaniel et. al [12]. The speed of the flow along the centerline is compared next. The comparison is shown in Fig. 14(b) along with theoretical predictions from Ashkenas \& Sherman [13]. The acceleration of the flow is properly captured, but the location of the shock is slightly ahead of the experiment.

Further comparisons are made for case 2 in Figs. 15(a) and 15(b). In this case, the higher pressure ratio located the shock further downstream then case 1 and a larger plume is obtained. The pressure and temperature along the centerline agree well with the theory of Ashkenas \& Sherman. The results also agree closely to the experiment and compare well to the viscous simulations by McDaniel et. al [12]. 


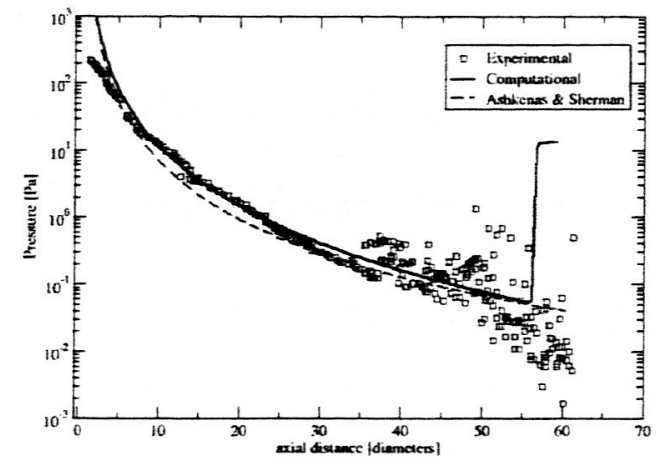

(a) Pressure along the centerline

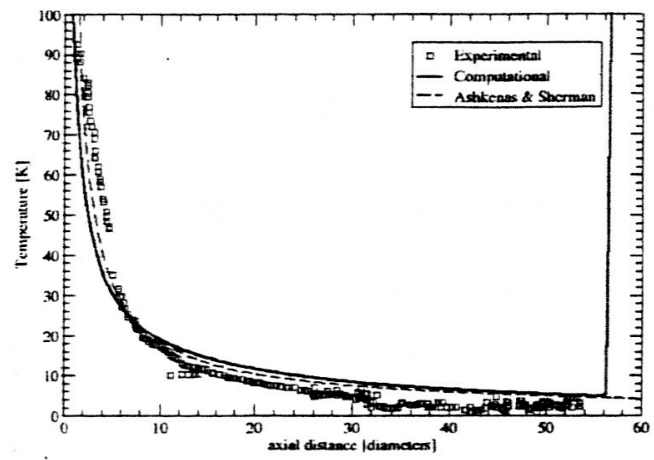

(b) Temperature along the centerline

Figure 15. Pressure and temperature comparisons for an under-expanded free jet (case 2)

\section{G. Space Shuttle}

During the STS-107 accident investigation, coupled 6-DOF simulations of debris emanating from the Space Shuttle Launch Vehicle (SSLV) during ascent were performed using Cartesian methods[14]. In these simulations the plumes emanating from the shuttle main engines (SSME) and solid rocket boosters (SRB) were ignored. While the plume effects had little effect on the flowfield ahead of the wing leading edge, and hence were not critical for the debris simulations during the accident investigation, the current method of simulating the engine plumes does provide higher-fidelity with little additional complexity. The plume exhausts on the shuttle are underexpanded, and hence do have a large effect on the flowfield and aerodynamic loads near the aft end of the vehicle. This is especially true for the bluff aft bodies of the shuttle orbiter and external tank. By ignoring the plumes the flow separates around these bluff bodies leading to large-scale unsteadiness. The plumes entrain much of this separated flow, leading to less flow unsteadiness and load excursions. Figure 16 show an example calculation of the SSLV flowfield at Mach 2.5 and $0.0^{\circ}$ angle of attack, with the SSME and SRB plumes operating at flight conditions. The Mach number contours on the body and centerline of the SSLV are shown, along with the plumes (blue is low, red is high). The shock waves are well resolved, and the low speed flow on the aft end of the orbiter and external tank is apparent. The high Mach number core of each plume is visible within the the SSME and SRB bell nozzles. The SSME and SRB nozzles are capable of gimbaling in this component geometry, and a companion paper[15] contains an automated parametric study of the SSLV flowfield including the effect of engine gimbal angle. This provides an automated, efficient method of simulating the complete SSLV geometry in any flight configuration. This ability can be used to provide high-fidelity, rapid-response,launch-day simulations, or other time-critical analysis.

\section{Concluding remarks}

A boundary condition based on flux update through the solution of a Riemann problem is presented within a Cartesian method. The procedure is shown to be an accurate engineering tool to model inlet flows and exhaust plumes on aircraft, spacecraft and missiles. The additional boundary model has no effect on robustness, or convergence of the multigrid algorithm and the CFL limitations are unchanged.

Several cases are presented for verification and validation. Two supersonic inlet designs that have subsonic flow in the inlet duct are compared to analytic solutions to validate the method for inlet flows. The Pitot inlet and the two-shock wedge-shaped inlet show that the method accurately predicts the strength and location 


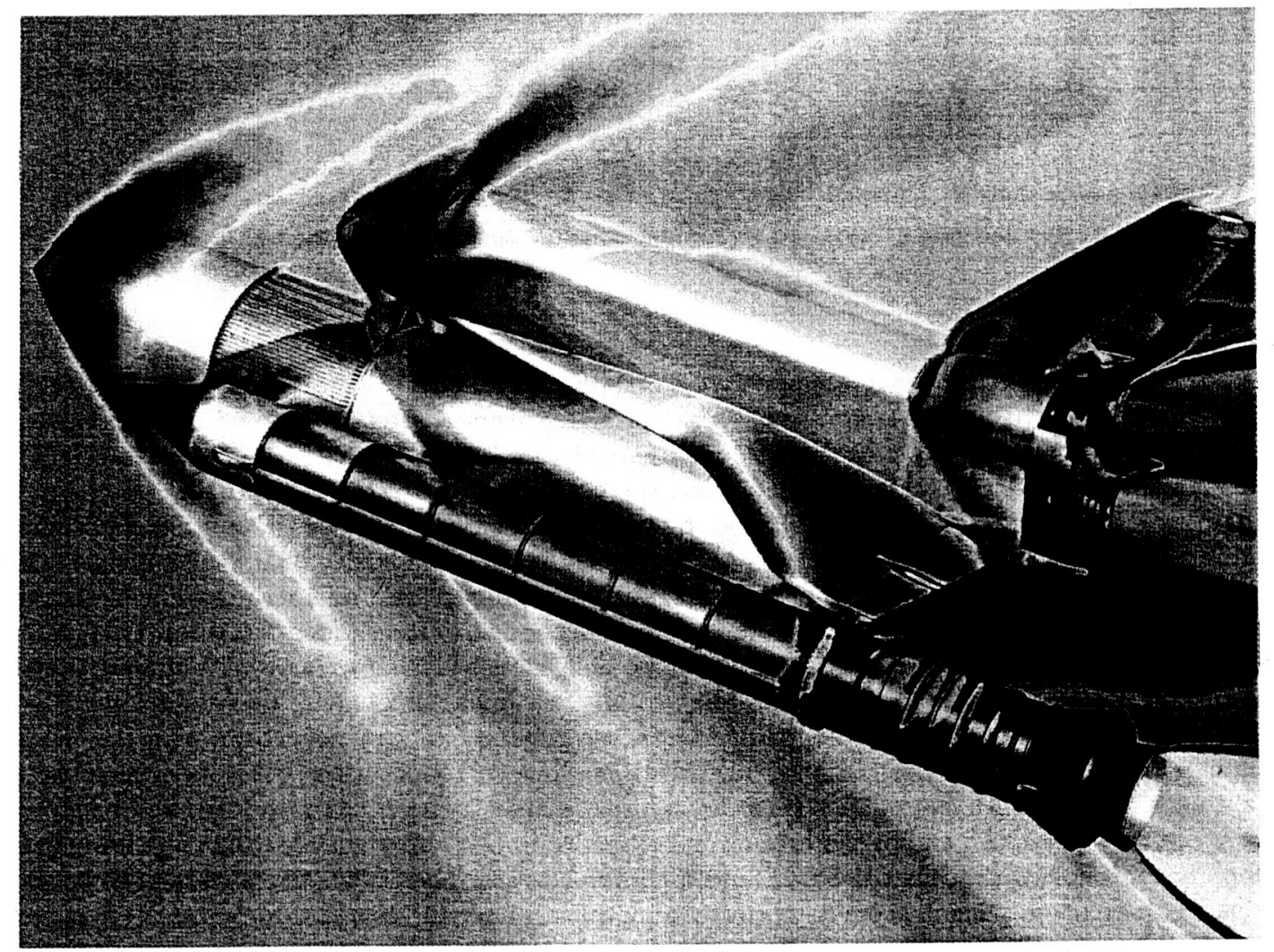

Figure 16. Mach number contours on the Space Shuttle in ascent at $M_{\infty}=2.5$ 
of a system of oblique and normal shocks based on user-specified conditions inside the inlet duct. The computational results compare extremely well with the gas dynamics theory and thus validate the capability for accurately modeling complex inlet flow and shock patterns given the conditions inside an inlet duct.

Two exhaust plume cases are compared to experiment to validate the exhaust capability of the method. The FESTIP test case shows that the size and shape of the plume as well as its effects on the flow field are well predicted. A simple ogive-shaped missile is simulated with exhaust and the pressures on the after-body are compared to an experiment for various chamber pressures which result in different plume sizes. It is concluded that the modeling of the nozzle internal geometry is preferable in order to accurately predict the sonic disk and ultimately the plume shape.

The inlet/exit boundary conditions are also applied to cases of practical interest with complex geometry. The inlet modeling of an F-16 fighter aircraft shows dramatic effect on the local flow in the vicinty of the inlet cowl. The exhaust from the rocket engines of a Space Shuttle Launch Vehicle (SSLV) is presented demonstrating the usefulness of the simple engineering method presented for a non-expert user to specify inlets and exhausts to obtain proper modeling of their effects.

The usefulness of this boundary conditions infrastructure can be enhanced by allowing the user to mimic a pressure jump at specified locations in the flow field instead of simply specifying a state. Such an "actuatordisk" type boundary condition would simplify modeling of fans, ducted fans, and helicopter rotors. Furthermore, the viscous layer close to the wall can have a substantial effect on the forces and moments. To obtain the gross effects of viscosity, this method can be extended with a boundary layer model to accurately capture viscous/inviscid interaction. Finally, the present model is validated for cold gas with no chemical reactions. Species modeling is necessary to extend the method to treat hot gas and chemically reacting flows.

\section{Acknowledgment}

The authors wish to thank Mike Olsen and Dr. William Chan of NASA Ames for their invaluable input during the course of this work. The authors would also like to thank Dr. M. Berger of NYU for useful discussions and Nick Georgiadis of NASA Glenn Research Center for help in obtaining experimental data and configurations. Dr. Murman was supported by NASA Ames Research Center (contract NAS2-00062) during this work.

\section{References}

${ }^{1}$ Flores, J., Chaderjian, N., and Sorenson, R., "Simulation of Transonic Viscous Flow over a Fighter-like Configuration Including Inlet," AIAA paper 87-1199.

${ }^{2}$ McGhee, R. J., "Jet-Induced Flow Separation on a Lifting Entry Body at Mach Number from 4.00 to 6.00," NASA Technical Memorandum NASA TM X-1997, NASA Langley Research Center, April 1970.

${ }^{3}$ DalBello, T., Georgiadis, N. J., Yoder, D. A., and Keith, T. G., "Computational Study of Axisymmetric Off-design Nozzle Flows," AIAA paper 2004-0530.

${ }^{4}$ Aftosmis, M. J., Berger, M. J., and Melton, J. E., "Robust and efficient Cartesian mesh generation for component-based geometry," AIAA Paper 97-0196.

${ }^{5}$ Aftosmis, M. J., Berger, M. J., and Adomovicius, G., "A parallel multilevel method for adaptively refined Cartesian grids with embedded boundaries," AIAA Paper 2000-0808.

${ }^{6}$ Aftosmis, M. J., Berger, M. J., and Adomovicius, G., "Parallel multigrid on Cartesian meshes with complex geometry," Proc. of the 8th Intl. Conf. on Parallel CFD, Trondhiem Norway, June 2000.

7 Bannink, W. J., Houtman, E. M., and Bakker, P. G., "Base Flow / Underexpanded Exhaust Plume Interaction in a Supersonic External Flow," AIAA paper 98-1598. 
8 Burt, J. R., "An Investigation of the Effectiveness of Several Devices in Simulating a Rocket Plume at Free Stream Mach Numbers of 0.9 to 1.2," Tech. Rep. RD-TR-71-22, U. S. Army Missile Command, Redstone Arsenal, Alabama, September 1971.

9 Staff, A. R., "Equations, Tables, and Charts for Compressible Flow," Tech. rep., Ames Aeronautical Laboratory, Moffett Field, CA, 1953.

10 Huband, G. W., Shang, J. S., and Aftosmis, M. J., "Numerical Simulation of an F-16A at Angle of Attack," J. of Aircraft, Vol. 27, No. 10, October 1990, pp. 886-892.

11 Raghunathan, S., Kim, H. D., Benard, E., Malon, P., and Harrison, R., "Plume Interference Effects on Missile Bodies," J. Spacecraft, Vol. 40, No. 1, 2002, pp. 136-138.

12 McDaniel, J. C. J., Glass, C. E., Staack, D., and Miller, C. G. I., "Experimental and Computational Comparison of an Underexpanded Jet Flowfield," AIAA Paper 2002-0305.

13 Ashkenas, H. and Sherman, F., "The Structure and Utilization of Supersonic Free Jets in Low Density Wind Tunnels," Rarefied Gas Dynamics, edited by J. H. de Leeuw, Vol. II, Supplement 3 of Advances in Applied Mechanics, Academic Press, 1966, pp. 84-105, Section 7: Experimental Methods in Rarefied Gas Dynamics.

${ }^{14}$ Gomez, R., Vicker, D., Rogers, S., Aftosmis, M., Chan, W., and Meakin, R., "STS-107 Investigation Ascent CFD Support," AIAA paper 2004-2226.

15 Murman, S., Aftosmis, A., and Nemec, M., "Parameter Studies Using a Cartesian Method," AIAA paper 2004-5076. 